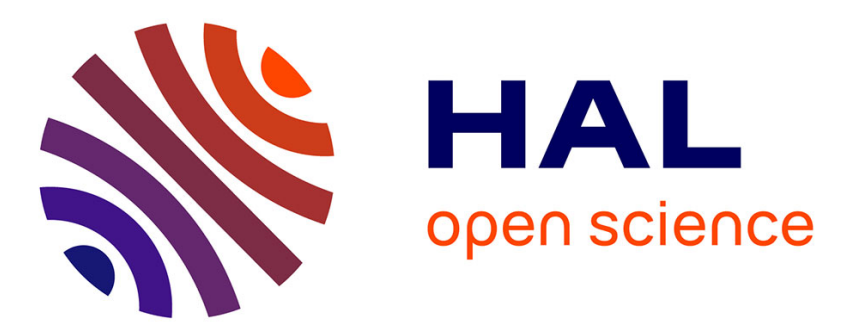

\title{
Des lésions cutanées diffuses
}

S. Ludwig-Béal, A. Camus, L. Popitean, L. Lévêque, Patrick Manckoundia

\section{To cite this version:}

S. Ludwig-Béal, A. Camus, L. Popitean, L. Lévêque, Patrick Manckoundia. Des lésions cutanées diffuses. La Revue de Médecine Interne, 2015, 36 (10), pp.712-714. 10.1016/j.revmed.2015.03.012 . hal-01295754

\section{HAL Id: hal-01295754 https://u-bourgogne.hal.science/hal-01295754}

Submitted on 25 Oct 2021

HAL is a multi-disciplinary open access archive for the deposit and dissemination of scientific research documents, whether they are published or not. The documents may come from teaching and research institutions in France or abroad, or from public or private research centers.
L'archive ouverte pluridisciplinaire HAL, est destinée au dépôt et à la diffusion de documents scientifiques de niveau recherche, publiés ou non, émanant des établissements d'enseignement et de recherche français ou étrangers, des laboratoires publics ou privés.

\section{다)(1) $(5$}

Distributed under a Creative Commons Attribution - NonCommercial| 4.0 International 
Image

\title{
Des lésions cutanées diffuses
}

\section{Diffuse cutaneous lesions}

\author{
F.-A. Wanvoegbe*, A. Turcu, L. Lévêque, H. Devilliers, B. Bach, G. Muller, P. Bielefeld, \\ J.-F. Besancenot
}

Service de médecine interne et maladies systémiques, CHU Dijon Bourgogne, 14, rue Paul-Gaffarel, 21000 Dijon, France

\section{N F O A A R T I C L E}

Historique de l'article :

Disponible sur Internet le 14 octobre 2018

\section{Mots clés :}

Panniculite

Sarcoïdose

Tomographie par émission de positons

\section{Keywords:}

Panniculitis

Sarcoidosis

Positron emission tomography

\section{L'histoire}

Une femme, âgée de 74 ans, consultait pour des lésions dermohypodermiques infiltrées des deux avant-bras, de couleur violacée, douloureuses au contact, s'étendant en regard de la partie externe des deux avant-bras, sur $5 \times 30 \mathrm{~cm}$ à gauche et $5 \times 5 \mathrm{~cm}$ à droite (Fig. 1A). Ces lésions avaient débuté quatre mois auparavant à gauche avec une extension progressive et une bilatéralisation après deux mois d'évolution. Il existait au niveau du membre inférieur gauche une modification de cicatrices anciennes contemporaine de l'apparition des lésions des membres supérieurs (Fig. 1B). La patiente signalait également un épisode récent de douleurs des épaules et de tuméfaction inflammatoire du poignet gauche, d'évolution favorable sous piroxicam. Par ailleurs, il n'y avait pas de myalgie ni d'acrosyndrome. Dans ses antécédents, on notait une surdité ancienne, une hernie discale opérée en 1989 avec arthrodèse en 2012, une HTA contrôlée par valsartan, une hypercholestérolémie traitée par simvastatine.

Il n'existait pas de syndrome inflammatoire, la numération formule sanguine montrait une lymphopénie à $830 / \mathrm{mm}^{3}$ sans hyperéosinophilie. La sérologie de Lyme était négative. La fonction

\footnotetext{
* Auteur correspondant.

Adresse e-mail : wafinarm@yahoo.fr (F.-A. Wanvoegbe).
}

rénale et la calcémie étaient normales. Les anticorps anti-nucléaires étaient positifs (1/320) avec une fluorescence membranaire, sans anti-ADN natif, ni anti-ENA. L'électrophorèse des protides et le complément étaient normaux. Le taux de l'enzyme de conversion de l'angiotensine était à $75 \mathrm{UI} / \mathrm{L}(\mathrm{N}<70)$. La biopsie de glandes salivaires accessoires ne révélait pas d'anomalie spécifique. Le QuantiFERON-TB Gold Plus ${ }^{\circledR}$ était négatif. Un TEP-scan était pratiqué (Fig. 2).

\section{Le diagnostic}

Une panniculite liée à une sarcoïdose systémique.

\section{Les commentaires}

Ces lésions faisaient évoquer en premier lieu une fasciite ou une panniculite dont les causes sont multiples : sclérodermie atypique, maladie de Lyme tertiaire avec acrodermatite atrophiante, syndrome de Schulman, sarcoïdose. La biopsie mettait en évidence des granulomes situés à la jonction dermo-hypodermique, composés d'éléments macrophagiques et de cellules géantes multinucléées cerclés par des éléments lymphocytaires, sans nécrose. Le scanner thoraco-abdomino-pelvien mettait en évidence des adénopathies hilaires bilatérales juxta-centimétriques non compressives.

Les manifestations cutanées de la sarcoïdose sont fréquentes, 25 à $35 \%$ des cas [1], mais il s'agit rarement de panniculites. Elles sont très polymorphes et classiquement séparées en lésions spécifiques, histologiquement granulomateuses, et non spécifiques (érythème noueux). Les manifestations cutanées secondaires à l'hypercalcémie sont exceptionnelles [2]. L'érythème noueux est caractérisé par un aspect histologique de panniculite non granulomateuse. Les lésions de panniculite granulomateuse se présentent souvent sous forme de plaques ovales ou annulaires, indurées, de couleur chair, érythémateuse ou brune. Les sites atteints comprennent les épaules et les bras, le dos et les fesses [3]. Les plaques étendues semblent en revanche très rares, voire exceptionnelles. Un cas de panniculite granulomateuse sarcoïdosique isolée a été rapporté par Gil et al. en 2002 chez une patiente de 59 ans, mais il s'agissait de nodules des membres inférieurs [4]. D'autres atteintes cutanées partagent des caractéristiques cliniques de la 

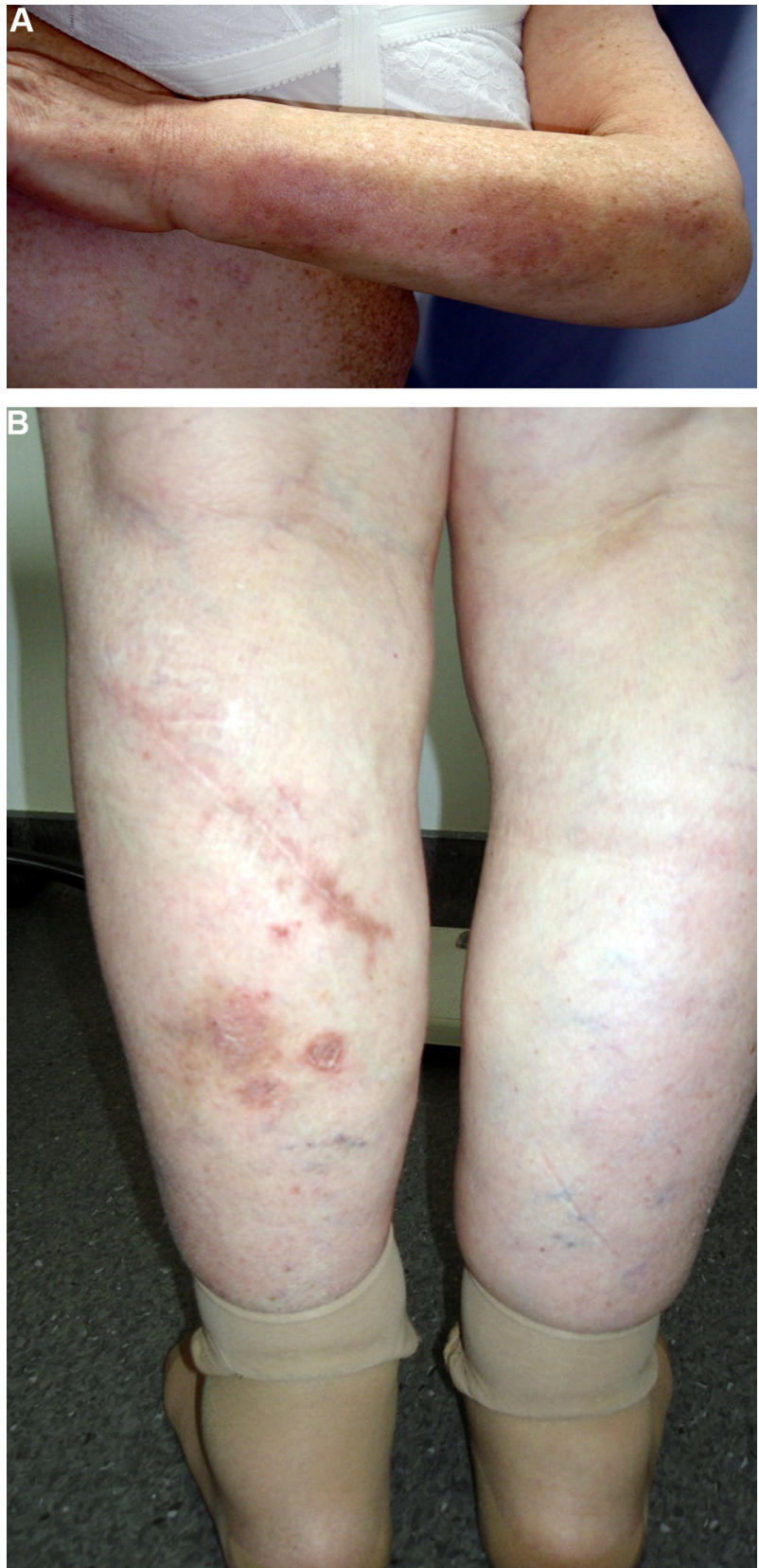

Fig. 1. Lésions infiltrées et étendues de l'avant-bras gauche (A), modifications de cicatrices anciennes (B).

sarcoïdose cutanée en placard et sont des diagnostics différentiels : psoriasis, lichen plan, lupus discoïde, granulome annulaire, lymphome cutané à cellules T, sarcome de Kaposi, syphilis secondaire [3]. Chez notre patiente, l'histologie montrait des lésions granulomateuses évocatrices d'une sarcoïdose cutanée, mais c'est le TEP-scan qui confirmait l'atteinte ganglionnaire médiastinale. Dans

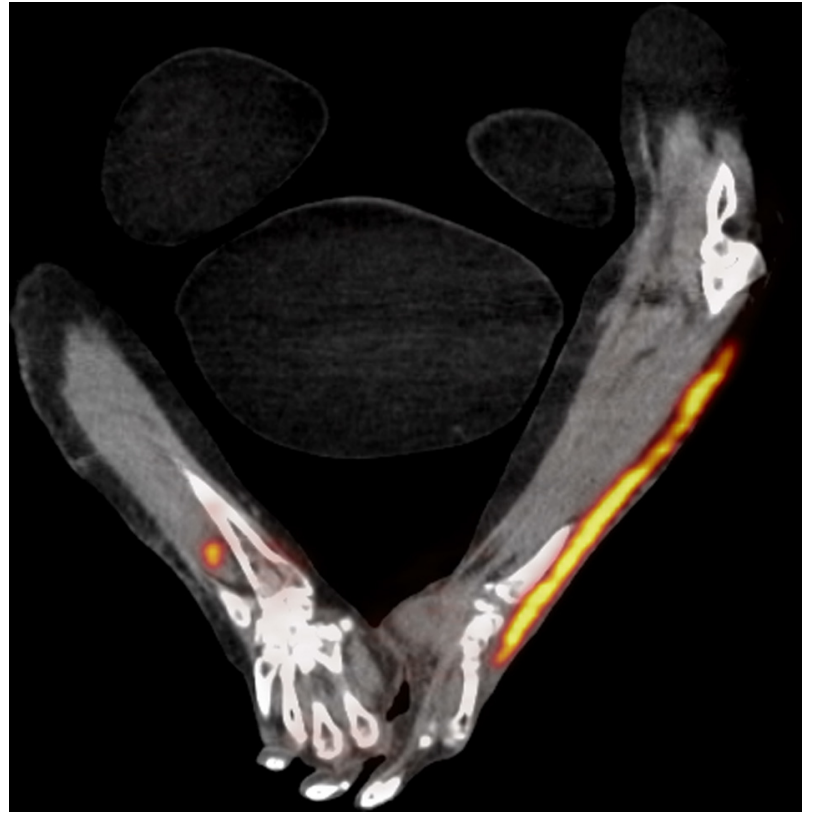

Fig. 2. TEP-scan : hypermétabolisme de l'avant-bras gauche.

la recherche diagnostique de la sarcoïdose, le TEP-scan a une sensibilité de $100 \%$ pour les lésions thoraciques ou naso-sinusiennes et de $80 \%$ pour les lésions pharyngo-laryngées [5]. La sensibilité globale pour l'ensemble des 36 localisations confirmées chez les 20 patients, était de $78 \%$ et de $87 \%$ après exclusion des atteintes cutanées potentiellement négatives en imagerie du fait de leur petite taille. Cette technique, réservée jusqu'alors aux localisations atypiques et aux formes évoluées et compliquées de la maladie, peut être envisagée à des stades plus précoces car elle peut révéler des localisations multiples et guider les biopsies indispensables au diagnostic.

\section{Déclaration de liens d'intérêts}

Les auteurs déclarent ne pas avoir de liens d'intérêts.

\section{Références}

[1] Descamps V, Bouscarat F, Marinho E. Manifestations cutanées de la sarcoïdose. EMC Dermatologie-Cosmétologie, 2. Paris: Elsevier Masson SAS; 2005. p. 177-88.

[2] Frieder J, Kivelevitch D, Menter A. Symptomatic hypercalcemia and scarring alopecia as presenting features of sarcoidosis. Proc (Bayl Univ Med Cent) 2018;31:224-6.

[3] Lodha S, Sanchez M, Prystowsky S. Sarcoidosis of the skin: a review for the pulmonologist. Chest 2009;136:583-96.

[4] Gil H, Mahammedi H, Hafsaouil C, Dupond AS, Meaux-Ruault N, Algros MP, et al. Panniculite granulomateuse sarcoïdosique isolée à propos d'un cas. Rev Med Interne 2002;23:645s.

[5] Braun JJ, Kessler R, Constantinesco A, Imperiale A. 18F-FDG PET/CT in sarcoidosis management: review and report of 20 cases. Eur J Nucl Med Mol Imaging 2008;35:1537-43. 